Pacific Journal of Mathematics

INTERSECTION REPRESENTATIONS OF GRAPHS BY ARCS 


\title{
INTERSECTION REPRESENTATIONS OF GRAPHS BY ARCS
}

\author{
Peter L. Renz
}

\begin{abstract}
In this paper we investigate relationship between the intersection pattern of families of arcs in a graph $H$ and the structure of $H$. Some steps are made toward an effective characterization of such patterns when $H$ is an acyclic graph.
\end{abstract}

An intersection representation of a graph $G$ is a function $r$ from $V(G)$, the set of vertices of $G$, into a family of sets $F$ such that distinct points $v_{1}$ and $v_{2}$ of $V(G)$ are neighbors in $G$ precisely when $r\left(v_{1}\right) \cap r\left(v_{2}\right) \neq \varnothing$. The graphs $G$ which admit intersection representations where the family $F$ is a family of intervals in a linearly ordered set have been characterized (Lekkerkerker and Boland [5], Fulkerson and Gross [2], and Gilmore and Hoffman [1]). A. C. Tucker [6] has made considerable progress in characterizing those graphs $G$ which admit intersection representations where $F$ is a family of ares in a circle. Our major concern here is the characterization of graphs which admit intersection representations where the family $F$ is a family of arcs in an acyclic graph, or tree. These graphs $G$ are characterized by Theorem 4.3 as being rigid circuit graphs which have the additional more complex property of normality defined below.

An arc is for our purposes either the empty set, a single vertex of a graph, or the set of edges and vertices of a simple path which includes its endpoints. This definition is the analogue of defining a topological arc to be either the empty set, a single point, or a set homeomorphic with the closed unit interval. The inclusion of the empty set and single points is a bit unusual but it will simplify some of the definitions and theorems which follow. The above makes clear what is meant when we say that $r$ is an intersection representation of a graph $G$ by arcs in a graph $H$. Such a representation $r$ is called semi-normal if $r(v) \cap r(w)$ is an arc in $H$ for all $v, w \in V(G)$. We say that $r$ is normal if $\cap\{r(v) \mid v \in S\}$ is a nonempty arc in $H$ whenever $S \subset V(G)$ spans a nonempty complete subgraph of $G$. A graph $G$ is called normal (semi-normal) if it admits a normal (semi-normal) intersection representation by arcs in some graph. Semi-normality and normality are basic assumptions of our approach. Our graphs will always be finite and free of loops and multiple edges. The main positive result proved is the following.

THEOREM 4.3. A graph $G$ has an intersection representation by 
arcs on an acyclic graph if and only if it is a normal rigid circuit graph.

The following argument leads to a useful remark on normal representations and acyclic graphs. Suppose that $H$ is an acyclic graph and $G$ has an intersection representation $p$ by paths in $H$. Arguing by using by Euler characteristic (see, for example, Lefshetz [4] and Klee [3]) one may prove that $p$ must be a normal representation. It is not difficult to see that if $H$ contains a cycle then some graph has a nonnormal intersection representation by arcs in $H$.

REMARK 1.1. A graph $H$ is acyclic if and only if every intersection representation by arcs in $H$ is normal.

2. Reduced normal representations. Soon we will restrict our attention to representations on acyclic graphs. We have shown that such representations have the virtue of being normal. We will say that $G$ is represented on $H$ if the $\operatorname{arcs}\{p(v) \mid v \in V(G)\}$ cover $H$. The virtue of a normal representation of $G$ on $H$ is that one may identify the maximal complete subgraphs (cliques) of $G$ with intervals in $H$ and then shrink these intervals in $H$ to points to obtain a new graph $H^{\prime}$. This shrinking induces a representation of $G$ on a graph $H^{\prime}$ having the property that the vertices of $H^{\prime}$ may be identified with the cliques in $G$. This reduction will enable us to restrict our attention to graphs whose vertices are cliques in $G$ and whose structure is closely related to that of $G$. The exact nature of this reduction will be made precise below.

A graph homeomorphism from the graph $H$ to the graph $H^{\prime}$ is a map $q$ of $V(H)$ into $V\left(H^{\prime}\right)$ with the following property. For distinct $k^{\prime}$ and $l^{\prime}$ in $V\left(H^{\prime}\right)$ there is an edge between $k^{\prime}$ and $l^{\prime}$ in $H^{\prime}$ if and only if there are $k$ and $l$ in $V(H)$ such that $k^{\prime}=q(k), l^{\prime}=q(l)$ and there is an edge between $k$ and $l$ in $V(H)$. A section graph of $a$ graph $H$ is a subgraph $K$ of $H$ such that the inclusion map from $K$ to $H$ is a graph homeomorphism. If $K$ is a section graph of $H$ then every graph homeomorphism of $H$ induces a natural graph homeomorphism on $K$. A graph homeomorphism $q$, as above, is connected if $q^{-1}$ of the vertices of a connected section graph $H^{\prime}$ always gives the vertex set of a connected section graph in $H$. A representation $p$ of $G$ on $H$ will be called simple if given $h \in V(H)$ there is a subset $S$ of $V(G)$ having the following property:

The connected component of $\cap\{p(s) \mid s \in S\}$

containing $h$ consists of $h$ alone. 
Notice that for a fixed $h \in V(H)$ and a fixed representation the union of sets $S$ possessing the above property also possesses the above property. Thus, if any such $S$ exist for $h$ and $r$, we define $S(h)$ to be the maximal set with the above property. Notice that $S(h)$ always spans a complete subgraph of $G$ whenever $S(h)$ is defined.

LeMMa 2.1. Let $p$ be a (semi-normal, normal) intersection representation of the graph $G$ by arcs on the graph $H$. Then there $i$ s a graph $H^{\prime}$ and a natural connected homeomorphism $q$ of $H$ onto $H^{\prime}$ such that $p q$ is a simple (semi-normal, normal) representation of $G$ on $H^{\prime}$.

We omit the proof of Lemma 2.1 which proceeds by straightforward induction on a cardinality of $V(H)$. This lemma leads to more complete results for normal representations. We give a few preliminary definitions. For a graph $G$ let $G^{*}$ be the family of all maximal complete subgraphs of $G$. Let $\mathscr{N}\left(G^{*}\right)$ be the nerve of $G^{*}$ (see, for instance, Lefshetz [4] for the topological notions of nerve and 1-skeleton).

Let $p, q, H, H^{\prime}$ and $G$ be as in Lemma 2.1. Let $p$ be normal and set $r=q p$. Then $r$ is normal and simple. The map $S$ is defined as above on $V\left(H^{\prime}\right)$ relative to $r$. It should be clear that $S(h)=\{s \in$ $V(G) \mid h \in r(s)\}$ for $h \in V\left(H^{\prime}\right)$. We show that this map gives an embedding of $H^{\prime}$ as a vertex spanning subgraph of the 1-skeleton of $\mathscr{N}^{*}\left(G^{*}\right)$. In showing this it is helpful to define $R(T)=\cap\{r(t) \mid t \in T\}$ for $T \subset$ $V(G)$. By the simplicity of $r, R(S(h))=h$ for $h \in V\left(H^{\prime}\right)$. Since $r$ is normal we then have $S(h) \in G^{*}$. Thus $S$ maps the vertices of $H^{\prime}$ into the vertices of $/ r\left(G^{*}\right)$. Since $R$ is an inverse for the mapping $S, S$ is injective. Let $T \in G^{*}$. Since $r$ is normal $R(T)$ is a nonempty arc in $H^{\prime}$. If $h$ is a vertex of $R(T)$ then $S(h) \supset T$ and $S(h)$ spans a complete subgraph in $G$. Hence $S(h)=T$ and $R(T)=h$ is a vertex of $H^{\prime}$. We note that this shows also $S(R(T))=S(h)=T$ so that $S$ is an inverse for $R$ and the maps are therefore bijections.

If $h$ and $k$ are neighbors in $H^{\prime}$, since $r$ represents $G$ on $H^{\prime}$, there is some $g \in V(G)$ such that $r(g)$ covers the edge in $H^{\prime}$ connecting $h$ and $k$. Thus $r(g) \in(S(h) \cap S(k))$ and hence there is an edge between $S(h)$ and $S(k)$ in the 1-skeleton of $\mathscr{N}\left(G^{*}\right)$. Thus $S$ provides a graph isomorphism of $H^{\prime}$ onto a subgraph $K$ of the 1-skeleton of $\mathscr{N}\left(G^{*}\right)$ such that $K$ includes all the vertices of $\mathscr{N}\left(G^{*}\right)$.

Consider the representation $S r$ of $G$ on $K$. The vertices of $K$ are the elements of $G^{*}$. For $T \in G^{*}$ and $v \in V(G)$ we have the following. If $v \in T$ then $R(T) \in r(v)$ so $T=S(R(T)) \in S(r(v))$. Conversely if $T \in$ $S(r(v))$ then $v \in T$ because every clique in $S(r(v))$ includes $v$. Thus $v \in T$ if and only if $T \in S(r(v))$. All of this leads to the following. 
COROLlaRY 2.2. Let $p$ be a normal intersection representation of the graph $G$ by arcs on the graph $H$. Then there is a connected graph homeomorphism $f$ of $H$ onto a subgraph $K$ of the 1-skeleton of $\mathscr{N}\left(G^{*}\right)$ such that

$K$.

(1) $f p$ is a normal intersection representation of $G$ by arcs in

(2) $K$ includes all of the vertices of $\mathscr{N}\left(G^{*}\right)$, and

(3) The vertices of $f p(v)$ are $\left\{T \in G^{*} \mid v \in T\right\}$ for all $v \in V(G)$.

The proof follows from the remarks above where we take $f=S q$ with $q$ as in Lemma 2.1.

It is worthwhile noticing that the construction of the homeomorphism $f$ in Corollary 2.2 is independent of arbitrary choices, although this has not been made explicit. The representation which any normal representation induces on a subgraph of the 1-skeleton of $\mathscr{N}\left(G^{*}\right)$ is the canonical reduced form for that representation.

3. Normal representations and the Euler characteristic. We will suppose that the graph $G$ has a normal representation $p$ on the graph $H$. Let $\mathscr{N}\left(G^{*}\right)$ be the nerve of $G^{*}$ and let $\chi$ denote the Euler characteristic (see, for instance, Lefshetz [4] for the topological notion of Euler characteristic and Klee [3] for the notion of a combinatorial Euler characteristic). Define $E(G)$ by $E(G)=\chi\left(\mathscr{N}\left(G^{*}\right)\right)$. Let $a_{0}$ be the number of connected components of $G$ (and therefore at $\mathscr{N}\left(G^{*}\right)$ ). If $H$ is thought of as a simplicial 1-complex embedded in $E^{3}$ we may use Klee's theory of the combinatorial Euler characteristic to compute $\chi(H)$ as below. We note that $\{p(v) \mid v \in V(G)\}$ is a cover of $H$. Furthermore, for any $S \subset V(G)$,

$$
\begin{aligned}
\chi(\cap\{p(s) \mid s \in S\}) & = \begin{cases}0 & \text { if } \cap\{p(s) \mid s \in S\} \text { is empty } \\
1 & \text { otherwise }\end{cases} \\
& = \begin{cases}0 & \text { if } S \text { does not span a complete subgraph of } G \\
1 & \text { otherwise }\end{cases} \\
& = \begin{cases}1 & \text { if } \varnothing \neq S \subset T \text { for some } T \in G^{*} \\
0 & \text { otherwise } .\end{cases}
\end{aligned}
$$

Let $|S|$ denote the cardinality of $S$. For $s \in V(G)$ let $s^{*}$ be the simplex in $\mathscr{N}\left(G^{*}\right)$ spanned by all $T$ in $G^{*}$ such that $s$ is in $T$. According to Klee [3] we may compute $\chi(H)$ as follows:

$$
\begin{aligned}
\chi(H) & =\sum(-1)^{|S|-1} \chi(\cap\{p(s) \mid s \in S\}) & & (\varnothing \neq S \subset V(G)) \\
& =\sum(-1)^{|S|-1} & & (S \subset V(G) \text { spans a complete }
\end{aligned}
$$




$$
\begin{array}{lll}
=\sum\left(-1^{|S|-1} \chi\left(\cap\left\{s^{*} \mid s \in S\right\}\right)\right) & & \text { subgraph in } G) \\
=\chi\left(\mathscr{N}\left(G^{*}\right)\right)=E(G) . & &
\end{array}
$$

The above relies upon the combinatorial properties of the Euler characteristic and finally upon the fact that $\left\{v^{*} \mid v \in V(G)\right\}$ is a well behaved cover of $\mathscr{N}\left(G^{*}\right)$ by simplices. This establishes the following.

THEOREM 3.1. If the graph $G$ has a normal representation on the graph $H$ then $E(G)=\chi(H)$.

In view of the known relationship of the Euler characteristic of a graph $H$ to the number of components of $H$, denoted $a_{0}(H)$, and the number of independent cycles in $H$, denoted $a_{1}(H)$ (see, for example, Lefshetz [4] p. 71) we have

$$
\begin{aligned}
\chi(H) & =a_{0}(H)-a_{1}(H) \\
& =a_{0}(G)-a_{1}(H) \\
& =E(G)
\end{aligned}
$$

where we use the fact $a_{0}(G)=a_{0}(H)$. These equations yield

$$
\begin{aligned}
& a_{0}(H)=a_{0}(G) \quad \text { and } \\
& a_{1}(H)=a_{0}(G)-E(G) .
\end{aligned}
$$

This shows how the Betti numbers of $H$ may be calculated from the structure of $G$.

The definition of a normal graph together with the above calculation and the characterization of acyclic graphs in terms of Betti numbers (Lefshetz [4]) lead to the following.

REMARK 3.2. A graph $G$ has in intersection representation by arcs on an acyclic graph if and only if $G$ is normal and $a_{0}(G)=E(G)$. In this case $G$ can admit normal representations only on acyclic graphs.

This remark reduces the problem of characterizing graphs with intersection representations by arcs on acyclic graphs to the problem of characterizing normal graphs such that $a_{0}(G)=E(G)$.

It is always possible to check a graph $G$ for normality by exhaustive search. By Corollary 2.2 it is sufficient to check whether $G$ admits a representation of the sort induced there on some subgraph $K$ of the 1-skeleton of $\mathscr{N}\left(G^{*}\right)$ (a spanning tree, if $G$ is rigid circuit). There are only finitely many possibilities. However, although it is possible to check many graphs easily by hand, no efficient algorithm for running such checks is known. Along this line one may ask if the global 
condition $E(G)=a_{0}(G)$ may be combined with some $k$-local condition on $G$ to insure that $G$ be normal, where by a $k$-local condition of $G$ we mean a condition involving sub-graphs of $G$ of some fixed maximum diameter $k$.

Some further remarks on graphs representable on acyclic graphs are worthwhile. A graph $G$ will be called locally linear if for each $g \in V(G)$ the graph spanned by $g$ and all of $g$ 's neighbors is representable on a line or linear graph. Every graph $G$ representable on an acyclic graph is easily seen to be locally linear. However, the graph $G_{0}$ (Figure 1) shows that local linearity plus $E\left(G_{0}\right)=a_{0}\left(G_{p}\right)$ does not imply normality.

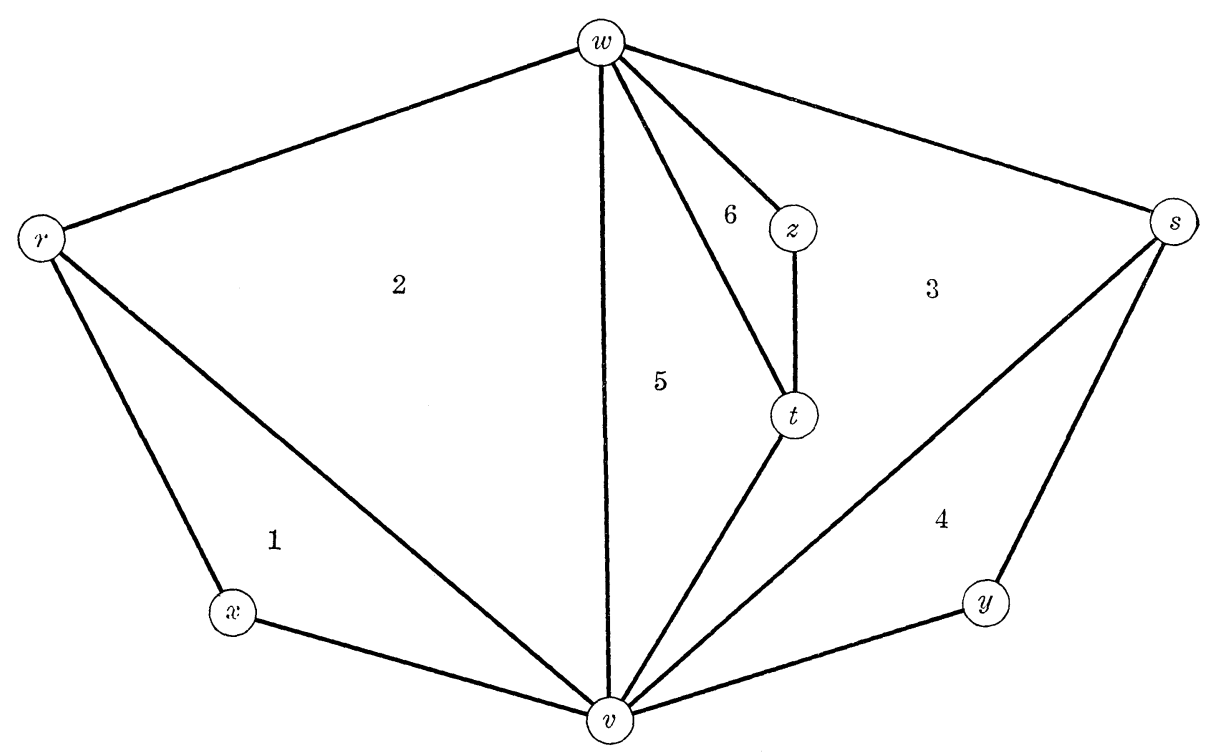

FIGURE 1.

The cliques of $G_{0}$ are numbered 1 through 6 .

The vertices of $G_{0}$ are labeled $r, s, t, v, w, x, y, z$.

In Figure 2 the cliques of $G_{0}$ are listed and given numbers. Any normal representation of $G_{0}$ leads via 2.2 and 2.3 to a representation on some subgraph $K$ of the 1 -skeleton of $\mathscr{N}\left(G_{0}^{*}\right)$. Let $p$ represent $G_{0}$ on $K$. If $u$ is a vertex of $G_{0}$ then the vertices in $K$ of $p(u)$ may be calculated using Remark 2.3. This information is also displayed in Figure 2.

If $G_{0}$ were normal then there is a graph structure $K$ for $G_{0}^{*}$ such that the relationships displayed in Figure 2 would be compatible with each of the paths mentioned there being simple. But we will show that no such graph structure for $G^{*}$ exists. Obviously $\{1,2\},\{3,4\}$, and $\{5,6\}$ would have to be edges of any such graph $K$. Since $p(v) \cap$ $p(w)$ is a sub-path of both $p(v)$ and $p(w), p(v) \cap p(w)$ must have one 


\begin{tabular}{c|c|c|c|c|c|c}
\hline Number & 1 & 2 & 3 & 4 & 5 & 6 \\
\hline $\begin{array}{l}\text { Vertex of } \\
\text { of (elements } \\
\text { of } G^{*} \text { ) }\end{array}$ & $\{r, x, v\}$ & $\{r, w, v\}$ & $\{s, w, v\}$ & $\{s, y, v\}$ & $\{w, t, v\}$ & $\{w, t, z\}$ \\
\hline
\end{tabular}

\begin{tabular}{l|l}
\hline \multicolumn{1}{c|}{ Path } & Vertices of path in $K$ \\
\hline$p(r)$ & $\{1,2\}$ \\
\hline$p(s)$ & $\{3,4\}$ \\
\hline$p(t)$ & $\{5,6\}$ \\
\hline$p(v)$ & $\{1,2,3,4,5\}$ \\
\hline$p(w)$ & $\{2,3,5,6\}$ \\
\hline$p(v) \cap p(w)$ & $\{2,3,5\}$ \\
\hline
\end{tabular}

FIGURE 2.
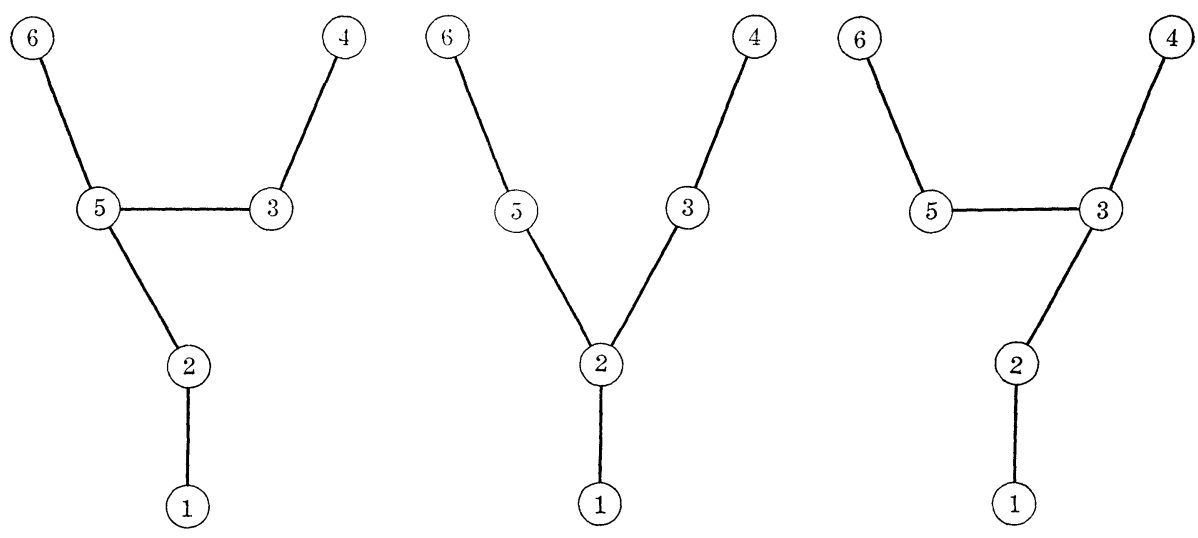

FIGURE 3.

of three possible forms. The possibilities for $K$ are then exactly as shown in Figure 3. None of them is compatible with the requirement that $p(v) \cap p(w)$ be a subpath of both $p(v)$ and $p(w)$.

It is not hard to see that $G_{0}$ is rigid circuit and locally linear. The above analysis should lend substance to the remarks following the introduction of the concept of a normal graph on how one may check a graph for normality.

4. Rigid circuit graphs. A graph $G$ is a rigid circuit graph if every cycle in $G$ has at least one triangular chord in $G$. Lekkerkerker and Boland [5] defined a simplicial vertex of a graph $G$ to be a vertex whose neighbors span a complete subgraph of $G$. They proved that every nonempty rigid circuit graph has a simplicial vertex. Fulkerson and Gross [2] gave the following characterization of rigid circuit graphs 
in terms of simplicial vertices. The graph $G$ is a rigid circuit graph if there is an enumeration $\left\{v_{i} \mid 1 \leqq i \leqq n\right\}$ of $V(G)$ such that $v_{i}$ is a simplicial point of the subgraph spanned by $\left\{v_{j} \mid 1 \leqq j \leqq i\right\}$ in $G$.

Using the Fulkerson and Gross characterization of rigid circuit graphs we may prove the following lemma.

LEMma 4.1. Let $C$ be a simplicial complex and let $p$ be an intersection representation of the graph $G$ by closed simplices of $C$ such that

(i) $\{p(g) \mid g \in V(G)\}$ is a cover of $C$ and,

(ii) whenever a nonempty set $S \subset V(G)$ spans a complete subgraph in $G$, then $\cap\{p(s) \mid s \in S\}$ is nonempty.

Then if $G$ is a connected rigid circuit graph $C$ is contractible over itself to a point.

We sketch the proof of this lemma. The proof is by induction on $k(G, C)=$ cardinality of $V(G)+$ cardinality of $V(C)$ where $V(C)$ is the vertex set of $C$. Since $G$ is a rigid circuit graph there is a simplicial vertex $v_{0}$ of $G$. We distinguish two cases. First, if $p\left(v_{0}\right)$ is a single point in $C$ we may suppress $v_{0}$ and $p$ will induce an intersection representation satisfying the hypotheses of Lemma 4.1 of the reduced graph on $C$. Thus we are finished in this case by induction. In the second case, if $p\left(v_{0}\right)$ is a nontrivial simplex in $C$ we construct a strong deformation retract $q$ of $C$ onto a proper subcomplex $C^{\prime}$ of $C$ such that $p$ followed by the retraction $q$ induces an intersection representation of $G$ on $C^{\prime}$ satisfying the hypotheses of Lemma 4.1. This strong deformation retract of $C$ may be constructed by collapsing two points of $\cap\left\{p(v) \mid v\right.$ and $v_{0}$ are neighbors in $\left.G\right\}$ to a point in $U\{p(v) \mid v$ and $v_{0}$ are neighbors in $\left.G\right\}$, leaving the other points of $C$ fixed. In the second case we finished by induction since $C$ has been retracted onto $C^{\prime}$ and $G$ is represented on $C^{\prime}$ with $V\left(C^{\prime}\right)<V(C)$. Thus by induction $C^{\prime}$ and hence $C$ is contractible over itself to a point.

Noticing that the natural intersection representation of a graph $G$ by simplices in $\mathscr{N}\left(G^{*}\right)$ satisfies the hypothesis of Lemma 4.1 we conclude that if $G$ is a connected rigid circuit graph $E(G)=\chi\left(\mathscr{N}\left(G^{*}\right)\right)=$ 1. Hence we have established:

TheOREM 4.2. If $G$ is a rigid circuit graph $E(G)=a_{0}(G)$.

The converse of this theorem is not true as the example $G_{1}$ (Figure 4) shows.

The remarks in $\S 3$ following the introduction of the concept of a normal graph together with the above lemma lead to a proof of 


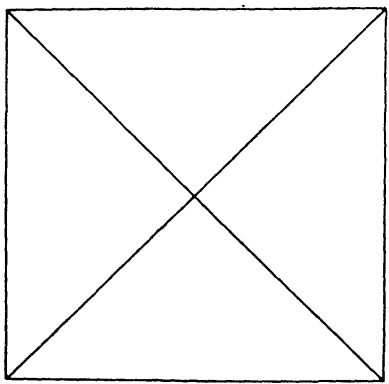

$G_{1}$

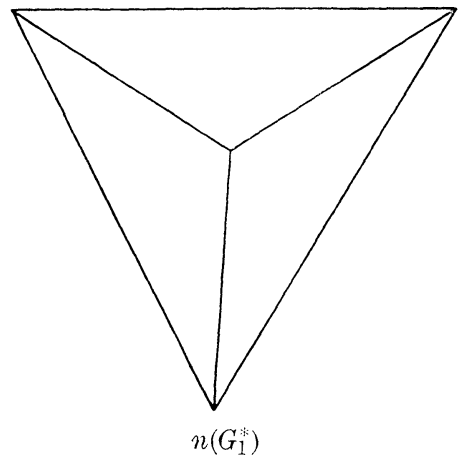

Figure 4.

$G_{1}$ is not rigid circuit but $N\left(G_{1}^{*}\right)$ is a tetrahedron so $E\left(G_{1}\right)=1$.

Theorem 4.3. First, the conditions are sufficient. If $G$ is rigid circuit and normal then by Remark $3.2 E(G)=a_{0}(G)$ and $G$ is normal. Thus $G$ may be represented on an acyclic graph. Second, the conditions are necessary. Without loss of generality we may assume that $G$ is represented on some acyclic graph. We will show that this implies that $G$ has a simplicial point. This will prove the sufficiency since every section graph of $G$ may also be represented on an acyclic graph and thus every nonempty section graph of $G$ must also have a simplicial point. Hence, by the criterion of Fulkerson and Gross, $G$ is a rigid circuit graph. We now prove that if a graph $G$ is representable on an acyclic graph then $G$ has a simplicial point.

In view of Corollary 2.2 and Remark 1.1 we may assume that vertices of $H$ are identified in the natural way with $G^{*}$. This reduction uses the fact that the graph homeomorphism of Corollary 2.2 preserves acyclicity of graphs. Since $H$ is acyclic it has at least one terminal vertex. Let $S \in G^{*}$ correspond to a terminal vertex $h_{0}$ of $H$. Then since $\cap\{p(s) \mid s \in S\}=h_{0}$ and since there is only one edge in $H$ emanating from the vertex $h_{0}$, there is an $s_{0} \in S$ such that $p\left(s_{p}\right)=h_{0}$. and $s_{0}$ is a simplicial point of $G$.

The effective algorithms which exist for finding linear representations of graphs (see [1] and [2]) suggest that one may search for normal representations (and hence representations on an acyclic graph) of rigid circuit graphs in an effective way by simply trying to knit the local representations together in a coherent way. However this approach merely suggests how such a calculation might proceed without givingdetails or suggesting the size of the problem. If a $k$-local condition guaranteeing normality were known the problem of checking a graph for normality would be considerably easier. Note that if such a condition works for rigid circuit graphs it would solve the problem of characterizing graphs representable on acyclic graphs. It would also probably lead quickly to an excluded subgraph characterization of such 
graphs. My conjecture is that no such $k$-local condition is sufficient and that further conditions of a global character are required.

Addendum. We give in Figure 5 another example of a locally linear nonnormal graph. Its pathology is sufficiently distinct from the earlier example to warrant inclusion.

The author wishes to thank Professor Victor L. Klee for suggesting this problem.

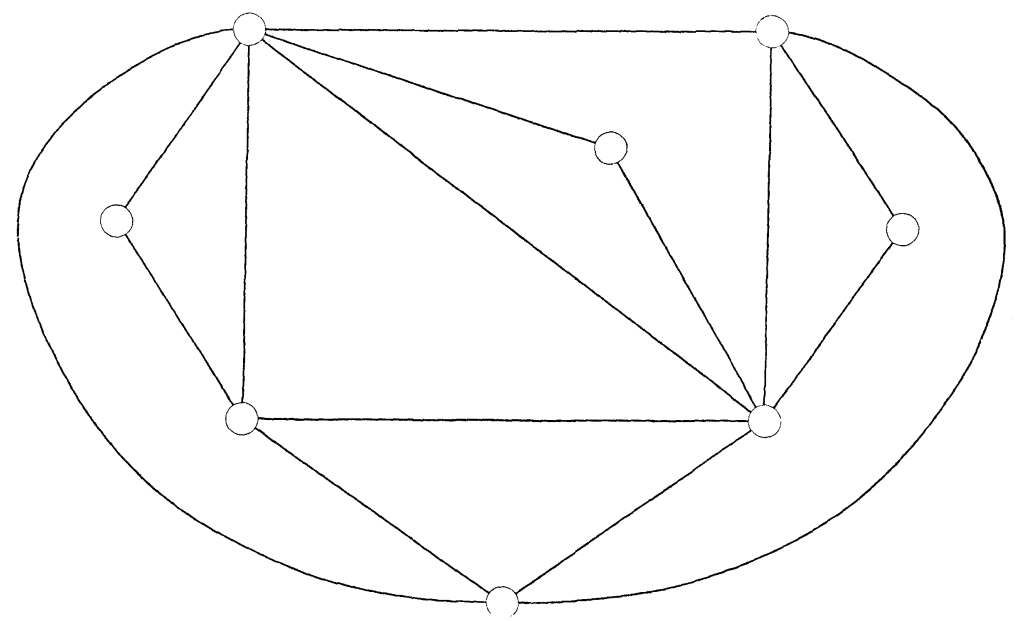

FIGURE 5.

\section{REFERENCES}

1. P. C. Gilmore and A. J. Hoffman, A characterization of comparability graphs and interval graphs, Canad. J. Math. 16 (1964), 539-548.

2. D. R. Fulkerson and O. A. Gross, Incidence matrices and interval graphs, Pacific

J. Math. 15 (1965), 835-855.

3. V. L. Klee, The Euler characteristic in combinatorial geometry, Amer. Math. Monthly 70 (1963), 119-127.

4. S. Lefshetz, Introduction to topology, Princeton University Press, Princeton, N.J., 1949.

5. C. G. Lekkerkerker and J. C. Boland, Representation of a finite graph by a set of intervals on the real line, Fund. Math. 51 (1962), 45-64.

6. A. C. Tucker, Ph. D. thesis, Stanford University, 1969.

Received February 24, 1970.

ReEd College

PORTLAND, OREGON 


\title{
PACIFIC JOURNAL OF MATHEMATICS
}

\author{
EDITORS
}

\author{
H. SAMELSON \\ Stanford University \\ Stanford, California 94305 \\ RichaRd PIERCE \\ University of Washington \\ Seattle, Washington 98105
}

J. DugundJI

Department of Mathematics

University of Southern California

Los Angeles, California 9.0007

RICHARD ARENS

University of California

Los Angeles, California 9.0024

\section{ASSOCIATE EDITORS}

E. F. BeCKenBACH
B. H. NeumanN

F. WoLE

K. YoSHIDA

\section{SUPPORTING INSTITUTIONS}

\author{
UNIVERSITY OF BRITISH COLUMBIA \\ CALIFORNIA INSTITUTE OF TECHNOLOGY \\ UNIVERSITY OF CALIFORNIA \\ MONTANA STATE UNIVERSITY \\ UNIVERSITY OF NEVADA \\ NEW MEXICO STATE UNIVERSITY \\ OREGON STATE UNIVERSITY \\ UNIVERSITY OF OREGON \\ OSAKA UNIVERSITY \\ UNIVERSITY OF SOUTHERN CALIFORNIA
}

\author{
STANFORD UNIVERSITY \\ UNIVERSITY OF TOKYO \\ UNIVERSITY OF UTAH \\ WASHINGTON STATE UNIVERSITY \\ UNIVERSITY OF WASHINGTON \\ AMERICAN MATHEMATICAL SOCIETY \\ CHEVRON RESEARCH CORPORATION \\ TRW SYSTEMS \\ NAVAL WEAPONS CENTER
}

The Supporting Institutions listed above contribute to the cost of publication of this Journal, but they are not owners or publishers and have no responsibility for its content or policies.

Mathematical papers intended for publication in the Pacific Journal of Mathematics should be in typed form or offset-reproduced, (not dittoed), double spaced with large margins. Underline Greek letters in red, German in green, and script in blue. The first paragraph or two must be capable of being used separately as a synopsis of the entire paper. The editorial "we" must not be used in the synopsis, and items of the bibliography should not be cited there unless absolutely necessary, in which case they must be identified by author and Journal, rather than by item number. Manuscripts, in duplicate if possible, may be sent to any one of the four editors. Please classify according to the scheme of Math. Rev. Index to Vol. 39. All other communications to the editors should be addressed to the managing editor, Richard Arens, University of California, Los Angeles, California, 90024.

50 reprints are provided free for each article; additional copies may be obtained at cost in multiples of 50 .

The Pacific Journal of Mathematics is published monthly. Effective with Volume 16 the price per volume (3 numbers) is $\$ 8.00$; single issues, $\$ 3.00$. Special price for current issues to individual faculty members of supporting institutions and to individual members of the American Mathematical Society: $\$ 4.00$ per volume; single issues $\$ 1.50$. Back numbers are available.

Subscriptions, orders for back numbers, and changes of address should be sent to Pacific Journal of Mathematics, 103 Highland Boulevard, Berkeley, California, 94708.

PUBLISHED BY PACIFIC JOURNAL OF MATHEMATICS, A NON-PROFIT CORPORATION

Printed at Kokusai Bunken Insatsusha (International Academic Printing Co., Ltd.), 7-17, Fujimi 2-chome, Chiyoda-ku, Tokyo, Japan. 


\section{Pacific Journal of Mathematics}

\section{Vol. 34, No. $2 \quad$ June, 1970}

Shair Ahmad, On the oscillation of solutions of a class of linear fourth order

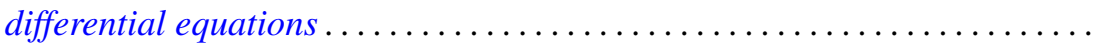

Leonard Asimow and Alan John Ellis, Facial decomposition of linearly

compact simplexes and separation of functions on cones ..............

Kirby Alan Baker and Albert Robert Stralka, Compact, distributive lattices of

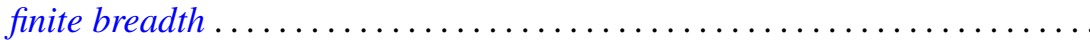

James W. Cannon, Sets which can be missed by side approximations to

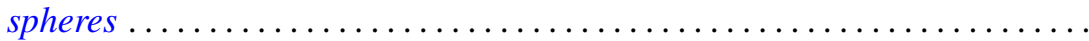

Prem Chandra, Absolute summability by Riesz means .................. 335

Francis T. Christoph, Free topological semigroups and embedding topological semigroups in topological groups....

Henry Bruce Cohen and Francis E. Sullivan, Projecting onto cycles in smooth, reflexive Banach spaces.................................

John Dauns, Power series semigroup rings .......................

Robert E. Dressler, A density which counts multiplicity ................

Kent Ralph Fuller, Primary rings and double centralizers ................

Gary Allen Gislason, On the existence question for a family of products.......

Alan Stuart Gleit, On the structure topology of simplex spaces .............

William R. Gordon and Marvin David Marcus, An analysis of equality in

certain matrix inequalities. $I \ldots \ldots \ldots \ldots \ldots \ldots \ldots \ldots$

Gerald William Johnson and David Lee Skoug, Operator-valued Feynman

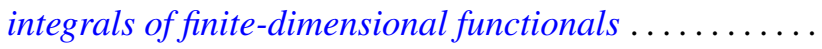

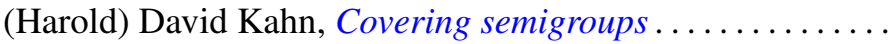

Keith Milo Kendig, Fibrations of analytic varieties

Norman Yeomans Luther, Weak denseness of nonatomic measures on perfect,

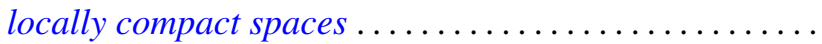

Guillermo Owen, The four-person constant-sum games; Discriminatory solutions on the main diagonal ...

Stephen Parrott, Unitary dilations for commuting contractions

Roy Martin Rakestraw, Extremal elements of the convex cone $A_{n}$ of

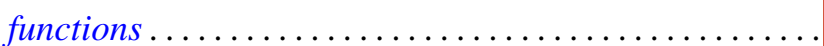

Peter Lewis Renz, Intersection representations of graphs by

William Henry Ruckle, Representation and series summability of complete

biorthogonal sequences.

F. Dennis Sentilles, The strict topology on bounded sets ...

Saharon Shelah, A note on Hanf numbers ...

Harold Simmons, The solution of a decision problem for several classes of rings. . .

Kenneth S. Williams, Finite transformation formulae involving the Legendre 